\title{
Electron Spin Resonance Study on Local Structure of Manganese lons Doped in Gamma-Aluminum Oxynitride Phosphors
}

\author{
Mamoru KITAURA*, Ai HARIMA*, Rong-Jun Xie**, Takashi TAKEDA**, Naoto HIROSAKI**, \\ Akimasa OHNISHI* and Minoru SASAKI*
}

*Yamagata University, Faculty of Science

**Nano Ceramics Center, National Institute of Material Science

Received May 27, 2011, Accepted July 27, 2011

\begin{abstract}
We have studied the local structure of manganese ion doped in gamma aluminum oxynitride phosphors ( $\gamma$-AION: Mn) using electron spin resonance (ESR) experiment. Six sharp peaks appear in ESR spectra of $\gamma$-AION: Mn, together with five weak doublets. These structures are caused by the hyperfine interaction of $3 d$ electrons with the ${ }^{55} \mathrm{Mn}$ nucleus of spin $l=5 / 2$. The six peaks are attributed to the allowed transitions $\left(\Delta M_{1}=0\right)$ between hyperfine splitting states of $M_{s}= \pm 1 / 2$. The five doublets are interpreted as the forbidden transitions $\left(\Delta M_{1}= \pm 1\right)$ between the hyperfine splitting states. The spectral feature of $\gamma$-AION: Mn resembles with the ESR spectrum of $\mathrm{Mn}^{2+}$ doped $\mathrm{MgAl}_{2} \mathrm{O}_{4}$ spinel, in which $\mathrm{Mn}^{2+}$ ions are substituted for tetrahedral $\mathrm{Mg}^{2+}$ sites. Since $\gamma$-AION is also of the spinel structure, it is suggested that $\mathrm{Mn}^{2+}$ ions occupy tetrahedral $\mathrm{Al}^{3+}$ sites. We further chased the effect of $\mathrm{Mg}^{2+}$ co-doping on ESR spectra of $\mathrm{Mn}^{2+}$ ions in $\gamma$-AION, in order to elucidate the luminescence enhancement in $y$-AION: $\mathrm{Mn}, \mathrm{Mg}$. It turned out that the $\mathrm{Mg}^{2+}$ co-doping increases $\mathrm{Mn}^{2+}$ ions at tetrahedral $\mathrm{Al}^{3+}$ sites.
\end{abstract}

KEYWORDS: oxynitride phosphor, $y$-AION, $\mathrm{Mn}^{2+}$ ion, electron spin resonance

\section{Introduction}

Transition-metal ions with $(3 d)^{\mathrm{n}}$ electron configurations have been utilized as activators in many phosphor materials, as well as rare-earth ions with $(4 f)^{\mathrm{n}}$ electron configurations. Among the transition-metal ions, the $\mathrm{Mn}^{2+}$ ion with five $3 d$ electrons is very attractive, because its luminescence shows high luminance and high chromatic purity. Actually, a few $\mathrm{Mn}^{2+}$ activated phosphors have been in practical use for display and solid state lightening ${ }^{1)}$. The luminescence properties of such phosphors strongly depend on ligands surrounding $\mathrm{Mn}^{2+}$ ions, and the luminescence wavelength can be controlled over the whole of the visible range by adjusting the lattice parameters of host materials ${ }^{2}$.

Recently, new types of inorganic phosphors have been developed using nitrides and oxynitrides as host materials. These phosphors have advantages of luminance, chromaticity and thermal quenching. The gamma aluminum oxynitride doped with $\mathrm{Mn}$ ions $(\gamma$-AlON: $\mathrm{Mn})$ is also one of such phosphors. Xie et al. have reported that this phosphor produces a green luminescence band at $512 \mathrm{~nm}$ under excitation with photons at $445 \mathrm{~nm}^{3}$. Furthermore, it has been also presented that the green luminescence band is enhanced by the addition of $\mathrm{Mg}^{2+}$ ions into $\gamma$-AlON: Mn. Since the $\mathrm{Mg}^{2+}$ codoped $\gamma$-AlON: Mn phosphor exhibits a small thermal quenching, high quantum efficiency and high color rendering, it has the potential to be a better green phosphor.

The host $\gamma$-AlON is the binary mixed compound of $\mathrm{Al}_{2} \mathrm{O}_{3}$ and $\mathrm{AlN}$, and is formed within the AlN concentration of $27-40 \mathrm{~mol} \%{ }^{4)}$. This material has a cubic spinel structure of the $F d 3 m$ space group, as same as $\mathrm{MgAl}_{2} \mathrm{O}_{4}$. Aluminum vacancies are inevitably introduced owing to the charge inconsistency between two different anions. There are two cation sites in the cubic spinel structure. One is the tetrahedral site coordinated by four anions. The other is the octahedral site coordinated by six anions. The point symmetry of the former and latter is $T_{d}$ and $O_{h}$, respectively. As generally known, a blue-green luminescence band appears when a $\mathrm{Mn}^{2+}$ ion is placed in the tetrahedral coordination, whereas an orange-red luminescence band appears when a $\mathrm{Mn}^{2+}$ ion is placed in the octahedral coordination $^{1)}$. Since a green luminescence band is strongly observed in $\gamma$-AlON: $\mathrm{Mn}$, it is expected that $\mathrm{Mn}^{2+}$ ions occupy tetrahedral $\mathrm{Al}^{3+}$ sites. On the other hand, Takeda et al. have investigated the X-ray absorption fine structure (XAFS) measurement of $\mathrm{Mn}-\mathrm{K}$ absorption in $\gamma$-AlON: $\mathrm{Mn}, \mathrm{Mg}^{5}$ ). From comparisons with the X-ray absorption near edge structure (XANES) of various manganese oxides, it was proved that the valence of the 
Mn ions was +2 . Furthermore, the curve fitting analysis of the extended X-ray absorption near edge structure (EXAFS) has revealed that $\mathrm{Mn}^{2+}$ and $\mathrm{Mg}^{2+}$ ions are predominantly placed at tetrahedral $\mathrm{Al}^{3+}$ sites. It was also pointed out that the point symmetry at the $\mathrm{Mn}^{2+}$ sites is lower than the $T_{d}$ symmetry.

The main aim of the present study is to obtain the information on the local structure of $\mathrm{Mn}^{2+}$ ions in $\gamma$-AlON through the analysis of magnetic interaction. Electron spin resonance (ESR) experiment was performed at room temperature using powder samples of $\gamma$-AlON: $\mathrm{Mn}$ and $\mathrm{Mg}^{2+}$ co-doped $\gamma$-AlON: Mn with different concentration of $\mathrm{Mn}^{2+}$ and $\mathrm{Mg}^{2+}$ ions. ESR spectra were analyzed using a spin-Hamiltonian, in order to determine spin-Hamiltonian parameters. The local structures of $\mathrm{Mn}^{2+}$ ions in $\gamma$-AlON: $\mathrm{Mn}$ and $\gamma$-AlON: $\mathrm{Mn}, \mathrm{Mg}$ are discussed in comparison with that in $\mathrm{MgAl}_{2} \mathrm{O}_{4}: \mathrm{Mn}$.

\section{Experimental}

Powder samples of $\gamma$-AlON: $\mathrm{Mn}$ and $\gamma$-AlON: $\mathrm{Mn}, \mathrm{Mg}$ were prepared by sintering the powder mixture of $a-\mathrm{Al}_{2} \mathrm{O}_{3}, \mathrm{AlN}, \mathrm{MgO}$, and $\mathrm{MnCO}_{3}$ at $1800^{\circ} \mathrm{C}$ for $2 \mathrm{~h}$ in the nitrogen atmosphere of $0.5 \mathrm{MPa}$. The concentration of $\mathrm{Mn}$ and $\mathrm{Mg}$ ions was adjusted to be $0.05-0.5 \mathrm{~mol} \%$ and 0.05-5 mol\%, respectively, in the preparation. The concentration of $\mathrm{Mn}$ ions is rather low, compared to that in Ref. 3. This is because the spectral analysis makes it possible to investigate the local structure of Mn ions. Since ESR spectra exhibit only a single peak in high Mn concentration, it is hard to obtain fruitful information on the local structure of $\mathrm{Mn}$ ions.

Powder X-ray diffraction (XRD) experiment was carried out to investigate whether phosphor powders synthesized have the crystal structure of $\gamma$-AlON. The characteristic X-ray from a copper target was used in

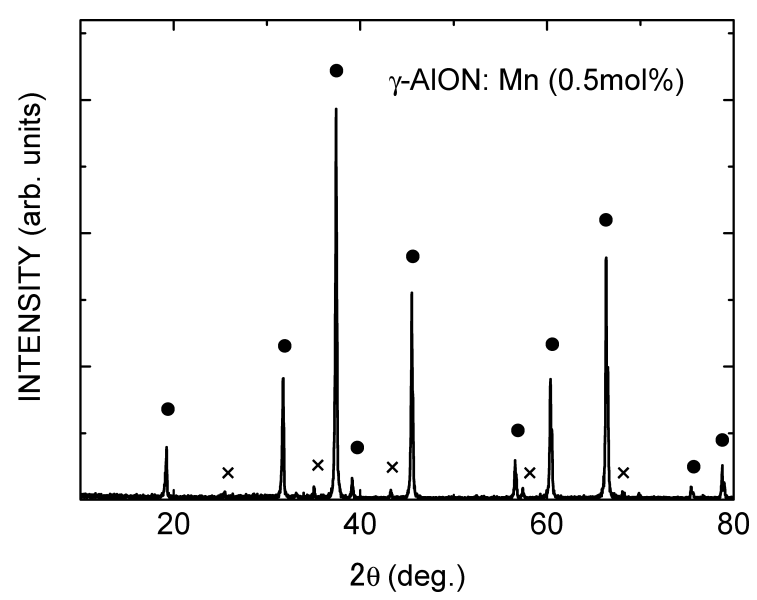

Figure 1 XRD spectrum of $y$-AION: $M n(0.5 \mathrm{~mol} \%)$ measured at room temperature. Solid circles correspond to XRD peaks of host $y$-AION reported in Ref. 3. A number of weak peaks are also seen as indicated by cross. They are due to the corundum phase of $\mathrm{Al}_{2} \mathrm{O}_{3}$. the XRD experiment. The result of $\gamma$-AlON: Mn $(0.5 \mathrm{~mol} \%)$ is shown as an example in Figure 1. The peak positions indicated by solids circles are good agreement with those of $\gamma$-AlON in Ref. 3, indicating that the phosphor powders prepared have the $\gamma$-AlON structure. Another peaks also appear in the XRD spectrum of Figure 1, as indicated by cross. These peaks are due to the corundum phase of $\mathrm{Al}_{2} \mathrm{O}_{3}{ }^{6}$. .

ESR measurement was performed at room temperature using a X-band ESR spectrometer (Joel, FA-600). ESR spectra were measured under the magnetic field modulation of $100 \mathrm{kHz}$. The microwave power was adjusted to be $0.5 \mathrm{~mW}$ during measurement, so as not to saturate ESR signals.

\section{Results and discussions}

The first and second derivatives of the ESR spectrum of $\gamma$-AlON: $\mathrm{Mn}(0.5 \mathrm{~mol} \%)$ are shown in the upper and lower parts of Figure 2, respectively. The positions of dips in the second derivative correspond to those of peaks in the original ESR spectrum which is not shown here. The original ESR spectrum consists of six prominent peaks and five weak doublets. The six peaks can be seen in inorganic phosphors doped with $\mathrm{Mn}^{2+}$ or $\mathrm{Mn}^{4+}$ ions. The possibility of $\mathrm{Mn}^{4+}$ ions excludes in $\gamma$-AlON: Mn, because this phosphor does not exhibit a red emission characteristic of $\mathrm{Mn}^{4+}$ ions ${ }^{1)}$, similar to that of $\mathrm{Cr}^{3+}$ ions of ruby.

A free $\mathrm{Mn}^{2+}$ ion has the ground state of ${ }^{6} S$ in the high spin state of $S=5 / 2$. The ${ }^{6} S$ state splits into six levels of

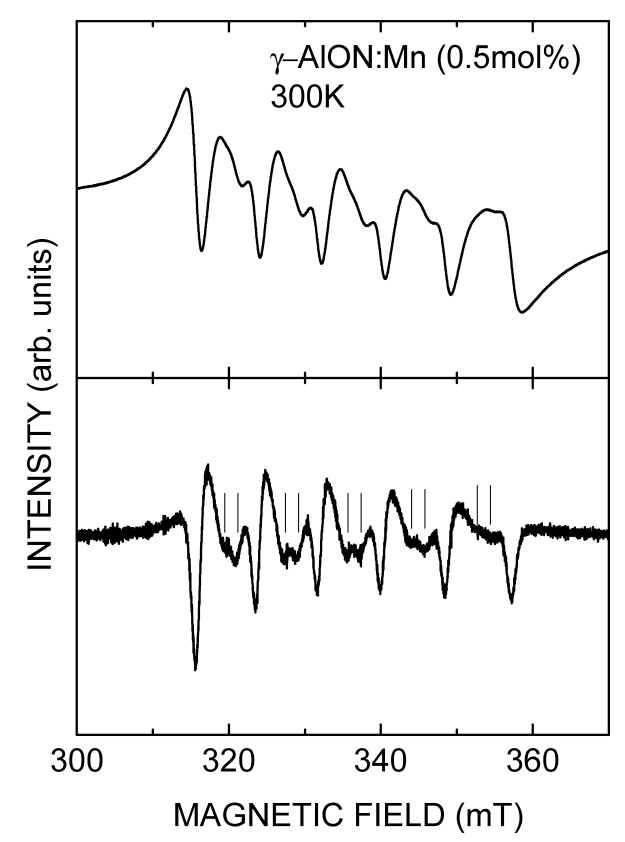

Figure 2 First (upper) and second (lower) derivative ESR spectra of $y$-AION: Mn (0.5 mol\%) measured at room temperature. Vertical bars indicate peak positions in the original ESR spectrum. The frequency of microwave was $9.438 \mathrm{GHz}$ during measurement. 
$M_{\mathrm{S}}= \pm 5 / 2, \pm 3 / 2, \pm 1 / 2$ by Zeeman splitting. In powder samples, the transition between $M_{\mathrm{S}}= \pm 1 / 2$ is only observable, because the peaks are remarkably enhanced by the superposition of ESR spectra from randomly oriented single crystallites with respect to an external magnetic field. The other transitions of $M_{\mathrm{S}}= \pm 5 / 2 \leftrightarrow \pm 3 / 2$ and $M_{\mathrm{S}}= \pm 3 / 2 \leftrightarrow \pm 1 / 2$ are not very conspicuous owing to the angular variation of their peaks. Each level of $M_{\mathrm{S}}= \pm 1 / 2$ further splits into six sublevels by the hyperfine interaction with the ${ }^{55} \mathrm{Mn}$ nucleus of spin $I=5 / 2$. The ESR transitions are allowed when the conditions of $\Delta M_{\mathrm{S}}= \pm 1$ and $\Delta M_{\mathrm{I}}=0$ are satisfied between initial and final states. Therefore, the six peaks are attributed to the transitions of $\Delta M_{\mathrm{I}}=0$ between the hyperfine splitting states of $M_{\mathrm{S}}= \pm 1 / 2$.

In order to determine the spin-Hamiltonian parameters, the spin-Hamiltonian of an axial symmetry is introduced by considering the symmetry lowering at $\mathrm{Mn}^{2+}$ sites pointed out in Ref. 5 . The formula is given by

$$
\mathcal{H}=g \mu_{\mathrm{B}} \boldsymbol{S} \cdot \boldsymbol{H}+D\left\{S_{D}^{2}-S(S+1) / 3\right\}+A \boldsymbol{S} \cdot \boldsymbol{I},
$$

where $g$ is the spectroscopic factor, $\mu_{\mathrm{B}}$ the Bohr magnetron, $D$ the zero-field splitting parameter of an axial symmetry, and $A$ the hyperfine structure coupling constant. The $z$ axis was chosen to be along the principal axis of symmetry. The parameter $D$ was set to be zero, because there was no fine structure due to the zerofield splitting term in Figure 2. The values of $g$ and $A$ were assumed to be isotropic. The energy eigenvalues were calculated up to the second order in perturbation $^{7}$. From the peak fit analysis of the six peaks, the values of $g$ and $A$ were determined to be 2.002 and $8.3 \mathrm{mT}$, respectively.

The ESR spectrum in the upper part of Figure 2 resembles that of $\mathrm{MgAl}_{2} \mathrm{O}_{4}: \mathrm{Mn}^{899}$. As listed in Table 1 , the values of $g$ and $A$ of $\gamma$-AlON:Mn are also good agreement with those of $\mathrm{MgAl}_{2} \mathrm{O}_{4}: \mathrm{Mn}^{10)}$. Especially, the agreement of the $A$ value suggests that there are similarities on the local structure of $\mathrm{Mn}^{2+}$ ions between these materials, because the spatial extension of $\mathrm{Mn}-3 d$ orbitals is reflected on the $A$ value. In $\mathrm{MgAl}_{2} \mathrm{O}_{4}: \mathrm{Mn}$, it was reported that $\mathrm{Mn}^{2+}$ ions occupy tetrahedral $\mathrm{Mg}^{2+}$ sites. We suppose that, in $\gamma$ - $\mathrm{AlON}: \mathrm{Mn}, \mathrm{Mn}^{2+}$ ions are placed at tetrahedral $\mathrm{Al}^{3+}$ sites. This supposition is supported by the fact that a green luminescence band is predominantly observed in $\gamma$-AlON: Mn.

In the lower part of Figure 2, one can see the five doublets, together with the six dips. Assuming that the doublets are caused by the transitions of $\Delta M_{\mathrm{S}}= \pm 1$ and $\Delta M_{\mathrm{I}}= \pm 1$, the positions are easily calculated using the spin-Hamiltonian parameters. The calculated result reproduces the positions of five doublets, as indicated by vertical bars. Therefore, it is reasonable to attribute the five doublets to the transitions of $\Delta M_{\mathrm{I}}= \pm 1$ between the
Table 1 Comparison of the spin-Hamiltonian parameters $g$ and $A$ between $\gamma$-AION: $\mathrm{Mn}$ and $\mathrm{MgAl}_{2} \mathrm{O}_{4}: \mathrm{Mn}$

\begin{tabular}{|l|c|c|}
\hline & $g$ & $A(\mathrm{mT})$ \\
\hline$\gamma$-AlON: $\mathrm{Mn}$ & 2.003 & 8.3 \\
\hline $\mathrm{MgAl}_{2} \mathrm{O}_{4}: \mathrm{Mn}$ & $1.998^{\mathrm{a}}, 2.003^{\mathrm{b}}$ & $8.2^{\mathrm{a}}, 8.3^{\mathrm{b}}$ \\
\hline
\end{tabular}

${ }^{a}$ Reference 8 .

${ }^{\text {b }}$ Reference 9 .

hyperfine splitting states of $\Delta M_{\mathrm{S}}= \pm 1 / 2$. The transitions are basically of ESR forbidden. The forbidden character is lifted through the admixture of hyperfine levels with the same $M_{\mathrm{S}}$ due to the third order perturbation terms containing the cross product of $D$ and $A^{11)}$. Accordingly, the parameter $D$ has to be a non-zero value, when the forbidden doublets appear in ESR spectra. In this case, many more peaks should be observed due to zero-field splitting. However, such peaks are not found in ESR spectra of Figure 2. This inconsistency would be explained by considering lattice distortions around $\mathrm{Mn}^{2+}$ ions. As we have already mentioned, the host $\gamma$-AlON contains two kinds of anions and $\mathrm{Al}^{3+}$ vacancies. Because of their inhomogeneous distributions, electrostatic interactions do not uniformly work on each of $\mathrm{Mn}^{2+}$ ions. Thus, various types of lattice distortions will be induced around $\mathrm{Mn}^{2+}$ ions, when they are introduced into $\gamma$-AlON. The superposition of ESR spectra of such $\mathrm{Mn}^{2+}$ ions will smear out many peaks due to the zerofield splitting. Therefore, it is likely that the ununiformity of lattice distortions around $\mathrm{Mn}^{2+}$ sites is responsible for $D=0$. This idea is in line with the result in Ref. 5 . Estimation of the zero-field splitting constant $D$ has been performed using the ESR spectrum of a powder sample ${ }^{7}$. This work may not be very meaningful in $\gamma$-AlON: Mn, because each of $\mathrm{Mn}^{2+}$ ions has the different $D$ value with each other.

To clarify the origin of the luminescence enhancement by the co-doping of $\mathrm{Mg}^{2+}$ ions, the relationship between the spin number and $\mathrm{Mg}^{2+}$ concentration was investigated. The result is shown in Figure 3. The spin number was estimated by integrating original ESR spectra. The value is proportional to the concentration of $\mathrm{Mn}^{2+}$ ions at tetrahedral $\mathrm{Al}^{3+}$ sites. In Figure 3, it is increased with the concentration of $\mathrm{Mg}^{2+}$ ions. Since the green luminescence band of $\gamma$ - $\mathrm{AlON}$ : $\mathrm{Mn}$ is also enhanced by the $\mathrm{Mg}^{2+}$ co-doping ${ }^{3)}$, it is natural that the luminescence enhancement is connected with the increase of the $\mathrm{Mn}^{2+}$ ions. In Ref. 3, it was revealed that the $\mathrm{Mg}^{2+}$ co-doping stimulates the formation of $\gamma$-AlON phase with a spinel structure of the $\mathrm{MgAl}_{2} \mathrm{O}_{4}$ type ${ }^{3)}$. In addition, it was also found that the lattice constant of $\mathrm{Mg}^{2+}$ co-doped $\gamma$-AlON: $\mathrm{Mn}$ is good agreement with that of $\mathrm{MgAl}_{2} \mathrm{O}_{4}$. Since the ionic radius of a $\mathrm{Mn}^{2+}$ ion is almost the same as that of $\mathrm{Mg}^{2+}$ ion ${ }^{12)}$, more $\mathrm{Mn}^{2+}$ ions 


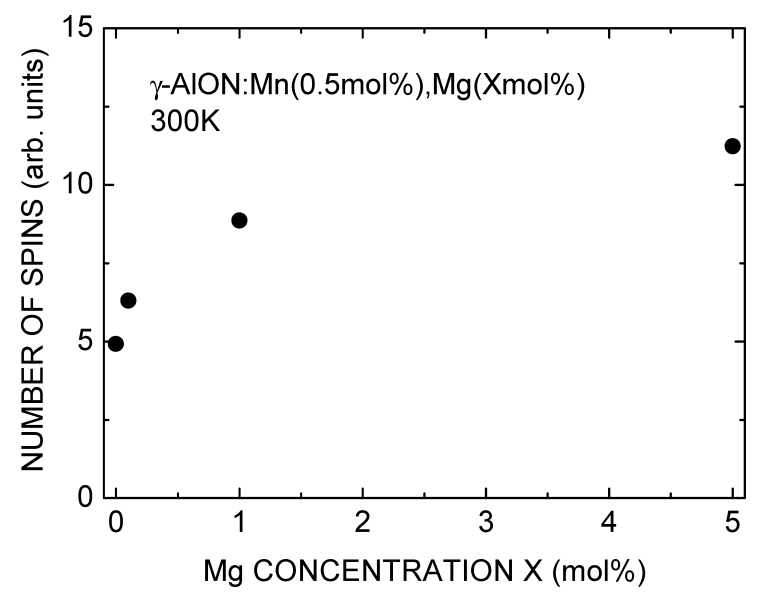

Figure 3 Relationship between the spin number and $\mathrm{Mg}^{2+}$ concentration in $\gamma$-AION: Mn, Mg. The spin number was estimated by integrating the original ESR spectra.

can be substituted for tetrahedral $\mathrm{Al}^{3+}$ sites by $\mathrm{Mg}^{2+}$ co-doing. Consequently, the increase of tetrahedral $\mathrm{Mn}^{2+}$ ions results in the luminescence enhancement of $\mathrm{Mg}^{2+}$ co-doped $\gamma$-AlON: $\mathrm{Mn}$.

\section{Summary}

In the present study, we have measured ESR spectra of $\gamma$-AlON: $\mathrm{Mn}$ and $\mathrm{Mg}^{2+}$ co-doped $\gamma$-AlON:Mn. The second derivatives of ESR spectra were composed of six prominent dips and five weak doublets. The former and latter were assigned to allowed transitions of $\Delta M_{\mathrm{S}}= \pm 1$ and $\Delta M_{\mathrm{I}}=0$ and forbidden transitions of $\Delta M_{\mathrm{S}}= \pm 1$ and $\Delta M_{\mathrm{I}}= \pm 1$. From the peak fit analysis, the spin-Hamiltonian parameters $g$ and $A$ were determined to be 2.002 and $8.3 \mathrm{mT}$, respectively. The values of $g$ and $A$ were good agreement with those of $\mathrm{MgAl}_{2} \mathrm{O}_{4}: \mathrm{Mn}$. It was thus suggested that $\mathrm{Mn}^{2+}$ ions doped in $\gamma$-AlON occupy tetrahedral $\mathrm{Al}^{3+}$ sites. An appearance of the five doublets was elucidated by the symmetry lowering at the $\mathrm{Mn}^{2+}$ sites. It was pointed out that the magnitude and/or direction of lattice distortions are not uniform at the $\mathrm{Mn}^{2+}$ sites. By adopting this idea, the absence of many peaks due to zero-field splitting was explained. Luminescence enhancement of $\mathrm{Mg}^{2+}$ co-doped $\gamma$-AlON : $\mathrm{Mn}$ was connected to the increase of tetrahedral $\mathrm{Mn}^{2+}$ ions.

\section{Acknowledgement}

This work was supported by Grant-in-Aid for Scientific Research (c) (No. 20613013) from Japan Society for the Promotion of Science. The authors acknowledge the Illuminating Engineering Institute of Japan for financial supports.

\section{References}

(1) Tamatani, S.: Phosphor Handbook, Yen, W. M., Shionoya, S., and Yamamoto, H., eds. CRC Press, Boca Raton, 2nd ed., Chap. 3 (2007).

(2) Kamimura, H., Sugano, S., and Tanabe, Y.: Ligand Field Theory and its Applications, Shokabo, Tokyo, 10th ed. (1990).

(3) Xie, R.-J., Hirosaki, N., Liu, X.-J., Takeda, T., and Li, H.-L.: Crystal Structure and Photoluminescence of $\mathrm{Mn}^{2+}, \mathrm{Mg}^{2+}$ Codoped Gamma Aluminum Oxynitride $(\gamma$-AlON): A Promising Green Phosphor for White Light-Emitting Diode, Appl. Phys. Lett., 92(20), p. 201905 (2008).

(4) McCauley, J. W., Patel, P., Chen, M., Gilde, G., Strassburger, E., Paliwal, B., Ramesh, K. T. and Dandekar, D. P.: Alon: A Brief History of its Emergence and Evolution, J. Euro. Ceram. Soc., 29(2), pp.223-236 (2009).

(5) Takeda, T., Horosaki, N., Xie, R-J., Matsushita, Y. and Homma, T.: Abstr. (2010 Annu. Meet., 2010) The Ceramic Society of Japan, 1J22 (in Japanese) (2010).

(6) For example, Brown, A. S., Spackman, M. A. and Hill, R. J.: The Electron Distribution in Corundum. A Study of the Utility of Merging Single-Crystal and Powder Diffraction Data, Acta Crystallogr. A, 49(3), pp. 513-527 (1993).

(7) Misra, S. K.: Estimation of the $\mathrm{Mn}^{2+}$ Zero-Field Splitting Parameter from a Polycrystalline EPR Spectrum, Physica B, 203(1-2), pp. 193-200 (1994).

(8) Tomita, A., Sato, T., Tanaka, K., Kawabe, Y., Shirai, M., Tanaka, K., and Hanamura, E.: Luminescence Channels of Manganese-Doped Spinel, J. Lumi., 109(1), pp. 19-24 (2004).

(9) Singh, V., Chakradhar, R. P. S., Rao, J. L., and Kim, D.-K.: Synthesis, Characterization, Photoluminescence and EPR Investigations of Mn Doped $\mathrm{MgAl}_{2} \mathrm{O}_{4}$ Phosphors, J. Solid State Chem., 180(7), pp. 2067-2074 (2007).

(10) Chiang, Y. and Kingery, W. D.: Grain-Boundary Migration in Non Stoichiometric Solid Solutions of Magnesium Alminate Spinel: I, Grain Growth Studies, J. Am. Ceram. Soc., Vol. 72(2), pp. 271-277 (1989).

(11) Abragam, A., and Bleaney, B.: in Electron Paramagnetic Resonance of Transition Ions, Clarendon Press, Oxford, Chap. 3 (1970).

(12) Shannon, R. D.: Revised Effective Ionic Radii and Systematic Studies of Interatomic Distances in Halides and Chalcogenides, Acta. Crystallogr. A, 30(5), pp. 751-767 (1976). 